\title{
ALGORITMO DE PREDICCIÓN DE ÍNDICES UV-B BASADO EN CASOS, MEDIANTE MEDICIONES DE MÍNIMA DISTANCIA EUCLIDIANA
}

\author{
UV-B INDEX PREDICTION ALGORITHM BASED ON CASES BY \\ MINIMUM EUCLIDEAN DISTANCE MEASUREMENTS
}

\author{
'Wilson Ferriol Mamani Yanapa; ' José Herrera Quispe
}

\begin{abstract}
RESUMEN
El presente trabajo propone un nuevo modelo de predicción de indices de radiación UV-B a partir de datos estadisticos anteriores. Esta propuesta extiende el modelo de proceso estocástico propuesto por Herrera (2013) donde se cambian los parámetros del componente deterministico por una función de similitud que usa la distancia euclidiana multidimensional ponderada y retardos de tiempo. Se adjunta un componente aleatorio heredado de un modelo Auto-regresivo de orden 1 . El modelo se aplica en la simulación de escenarios climáticos con altos indices de radiación UV-B. Se busca evaluar el porcentaje de respuesta de la radiación solar, además de validar la efectividad del algoritmo para datos proporcionados por la dirección regional de salud de Arequipa.
\end{abstract}

Palabras Clave: algoritmo de predicción, razonamiento basado en casos, radiación UV-B.

\section{ABSTRACT}

It is proposed a new model for predicting levels of $U V$-B radiation from previous statistical data. This proposal extends the model of stochastic process proposed by Herrera (2013) where the deterministic component parameters are changed by a function of similarity using the weighted multidimensional Euclidean distance and time delays; it is attached a random component inherited an Auto-regresivo model of order 1. The model is applied in the simulation of climate scenarios with high levels of UV-B radiation, to assess the response rate of solar radiation, as well as validate the effectiveness of the algorithm for data provided by the regional Directorate of Arequipa health.

Key Words: prediction algorithm, case-based reasoning, uv-b radiation

\section{INTRODUCCIÓN}

El agujero de la capa de ozono apareció en los años 80 sobre la Antártida durante las primaveras del hemisferio sur, debido a la acumulación de gases freón, los cuales están relacionados con la atmósfera a través de diversas actividades humanas [2]. La baja concentración de ozono en la capa correspondiente forma agujeros que permiten el ingreso de mayor cantidad de radiación ultravioleta de cortas longitudes de onda (llamadas UV-B) Los bordes del agujero abarca el sur del continente australiano, Nueva Zelanda y el continente sudamericano [3].

Durante las últimas décadas, las campañas públicas de salud han sido implementadas difundir los riesgos de la radiación UV. En una parte importante de los programas de prevención se recomienda el uso estricto de implementos de protección, con el objetivo de reducir el cáncer de piel. La protección solar es de particular importancia en distintos grupos de riesgo (pacientes con xeroderma pigmentosum, síndrome nevus de células básicas o receptores de trasplantes de órganos), quienes tienen alta probabilidad de desarrollar cáncer de piel.

Esto ejerce una significativa influencia en numerosos procesos que toman lugar en la atmósfera y la biósfera. La radiación UV afecta la salud humana (induce Eritema o quemadura de sol, daños en el ADN, cáncer de piel y cataratas, favorece la síntesis de vitamina D). Produce degradación de materiales (polímeros, pigmentos y tintes). Induce modificaciones en la química atmosférica y ciclos biogeoquímicos [5]; afectando plantas y ecosistemas terrestres [6]; fotosintesis impares en muchas especies de plantas e incrementa la susceptibilidad de las plantas a enfermedades [7], afectando el cultivo y desarrollo de cosechas [8], daños en ecosistemas acuáticos, reduce la productividad de fitoplancton e induce cambios en comunidades microbiológicas e invertebradas, los cuales pueden afectar el ciclo de nutrientes en los suelos [8].

La Organización Meteorológica Mundial (OMM), 
la Organización Mundial de la Salud (OMS) y el Programa de Naciones Unidas para el Medio Ambiente (PNUMA) han propuesto la creación de un índice simple que informa a la población del riesgo, este es el Índice Ultravioleta (IUV) que varía entre 0 a 16 y tienc cinco rangos: Bajo (1 a 2), moderado (3 a 4), alto (5 a 7), muy alto (8 a 10) y extremadamente alto (11 a ms) [9].

En adición, el análisis de varios parámetros de exposición solar ha demostrado contundentemente una asociación entre el desarrollo de melanomas malignos y las intensas y cortas exposiciones de radiación $\mathrm{UV}$, principalmente en la niñez [10].

Según el Ministerio de Salud del Perú, cada año son diagnosticados más de cuatro mil casos de cáncer de piel, debido, entre otros factores, a los elevados índices de radiación ultravioleta a los que están sobreexpuestas las personas.

El Servicio Nacional de Meteorología e Hidrología del Perú (SENAMHI), realiza monitoreo de la radiación UV$\mathrm{B}$ en diferentes ciudades del pais y publica diariamente el indice IUV [12].

Por ello es de vital importancia la implementación de modelos computacionales capaces de pronosticar los indices de radiación UV-B, a fin de estudiar el comportamiento y realizar acciones de prevención en la población local.

\section{MATERIALES Y MÉTODOS}

\subsection{Conceptualización sobre algoritmo de predicción UV-B}

\section{Algoritmo}

Es un conjunto prescrito de instrucciones o reglas bien definidas, ordenadas y finitas que permite realizar una actividad mediante pasos sucesivos que no generen dudas a quien deba realizar dicha actividad [14]

Muchos algoritmos son ideados para implementarse en un programa. Sin embargo, los algoritmos pueden ser implementados en otros medios, como una red neuronal, un circuito eléctrico o un aparato mecánico y eléctrico.

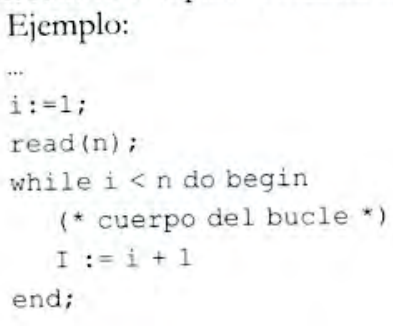

\section{Predicción}

El término predicción puede referirse tanto a la acción y al efecto de predecir [15] como a las palabras que manifiestan aquello que se predice; en este sentido, predecir algo es anunciar por revelación, ciencia o conjetura algo que ha de suceder [15].

La predicción constituye una de las esencias claves de la ciencia, de una teoría cientifica o de un modelo científico. Así, el éxito se mide por el éxito o acierto que tengan sus predicciones [16].

\section{Radiacion UT}

En el área de la fotobiología, se define como radiación ultravioleta al intervalo de radiaciones electromagnéticas menores a $400 \mathrm{~nm}$ [17]. La radiación ultravioleta se puede subdividir en cuatro intervalos:

- La que produce envejecimiento (aging) denominada UVA $(315 \mathrm{a} 400 \mathrm{~nm})$,

- La que produce quemadura (burning) denominada UVB $(280$ A $315 \mathrm{~nm})$,

- La que los autores denominan cosmic UV-C (200 a $280 \mathrm{~nm}$ ) y finalmente,

- La denominada "vacio" vacum (debido a que como es absorbida por el oxígeno y nitrógeno solo es posible que se propague en condiciones de vacío) y corresponde a fotones con longitud de onda menor a $200 \mathrm{~nm}$.

\subsection{Sistema basado en casos}

Un Sistema Basado en Casos para la toma de decisiones en un Sistema de Decisión $\mathrm{S}$ como se presenta en (1), y se define en términos de un par $(\mathrm{U}, \mathrm{X} \cup \mathrm{Y})$ donde $\mathrm{U}$ es un conjunto finito no vacío de objetos, eventos llamados casos, mientras X y Y son dos conjuntos finitos, no vacíos, de atributos o propiedades llamados rasgos predictores y objetivos respectivamente.

$$
\mathrm{S}=(\mathrm{U}, \mathrm{X} \cup \mathrm{Y})
$$

Cada rasgo predictor $\mathrm{x}_{\mathrm{i}} \in \mathrm{X}$ puede ser considerado una función, como la que aparece en (2), que mapea elementos de $\mathrm{U}$ en el conjunto $\mathrm{M}_{i}=\mathrm{x}_{i 1}, \mathrm{x}_{12}, \ldots, \mathrm{x}_{\mathrm{iq}}$, el cual se denomina conjunto de valores del rasgo predictor $\mathrm{X}_{\text {. }}$.

$$
\mathrm{x}_{i}: \mathrm{U} \rightarrow \mathrm{M}_{i}
$$

De mancra análoga cada rasgo objetivo $y_{i} \in \mathrm{Y}$ puede ser considerado una función que mapea elementos de $\mathrm{U}$ en el conjunto $\mathrm{N}_{i}=y_{1} 1, y_{i} 2, \ldots, y_{i} \theta$ ique se denomina conjunto de valores del rasgo predictor $y_{i}$. Dicha función puede verse en (3)

$$
\mathrm{y}_{\mathrm{i}}: \mathrm{U} \rightarrow \mathrm{N}_{\mathrm{i}}
$$

Las componentes de un Sistema Basado en Casos son: la base de conocimiento o base de casos, el módulo de recuperación de casos y el módulo de adaptación de las soluciones.

\section{Base de casos}

La base de casos contiene las experiencias o casos con los cuales el sistema realiza sus inferencias y puede ser representada a través de una tabla, cuyas columnas son etiquetadas por variables o atributos que representan los rasgos predictores y objetivos, mientras que sus filas representan los casos. En la tabla 1, se representa la estructura de la base de casos de nuestro problema. El conjunto $\mathrm{U}$ está formado por los casos $\mathrm{O}_{1}, \mathrm{O}_{2}, \ldots, \mathrm{O}_{\mathrm{m}}$; el conjunto $\mathrm{X}$ por los atributos $\mathrm{x}_{1}, \ldots \mathrm{x}_{\mathrm{n}}$, mientras que el conjunto $\mathrm{Y}$ lo integra el atributo $\mathrm{y}_{1}$. 
Mamani W, y Herrera J., Algoritmo de Predicción de Indices UV-B Basado en casos, mediante mediciones de Minima Distancia Euclidiana

Tabla $\mathrm{N}^{\circ} 01$. Representación de la estructura de la base de casos.

\begin{tabular}{ccc}
\hline & Rasgos predictores & Rasgo objetivo \\
\hline Casos & $\mathrm{x}_{1} \ldots \mathrm{x}_{\mathrm{n}}$ & $\mathrm{y}_{t}$ \\
$\mathrm{O}_{1}$ & $\mathrm{x}_{1}\left(\mathrm{O}_{t}\right) \ldots \mathrm{x}_{\mathrm{n}}\left(\mathrm{O}_{1}\right)$ & $\mathrm{y}_{1}\left(\mathrm{O}_{1}\right)$ \\
$\mathrm{O}_{2}$ & $\mathrm{x}_{1}\left(\mathrm{O}_{2}\right) \ldots \mathrm{x}_{\mathrm{n}}\left(\mathrm{O}_{2}\right)$ & $\mathrm{y}_{1}\left(\mathrm{O}_{2}\right)$ \\
$\ldots$ & $\ldots$ & $\ldots$ \\
$\mathrm{O}_{\mathrm{m}}$ & $\mathrm{x}_{1}\left(\mathrm{O}_{\mathrm{m}}\right) \ldots \mathrm{x}_{\mathrm{n}}\left(\mathrm{O}_{\mathrm{m}}\right)$ & $\mathrm{y}_{1}\left(\mathrm{O}_{\mathrm{m}}\right)$ \\
\hline
\end{tabular}

En nuestro problema, el conjunto $U$ estaría integrado por los 365 días del año estudiados; el conjunto $\mathrm{X}$ lo integrarian los indices de radiación registrados anteriormente (rasgos predictores), almacenados en una base de casos y el conjunto $Y$ estaría formado por los índices que siguen a un grupo de vector con rasgos predictores anteriores.

\section{Módulo de recuperación}

El proceso de recuperación consiste en determinar los casos de la base más sencilla, de ellas se cuenta el número de rasgos predictores similares entre ambos. Otra técnica utiliza un conjunto de heurísticas que determinan las variables de mayor importancia (peso) en la determinación del rasgo objetivo y se formula una función que teniendo en cuenta esto se considera la semejanza o diferencia entre cada uno de los rasgos predictores.

A continuación se describe el algoritmo del proceso de recuperación.

Entrada: $\mathrm{O}_{i}, \mathrm{O}_{t}\left(\mathrm{O}_{0}\right.$ : nuevo problema y $\mathrm{O}_{\mathrm{r}}$ : caso de la base). Salida: $\beta\left(\mathrm{O}_{i}, \mathrm{O}\right)$ (Medida de semejanza o distancia entre $\mathrm{O}_{0}$ y $\mathrm{O}_{\text {) }}$

Para cada caso $\mathrm{O}_{\mathrm{x}}$ se requiere:

i) Buscar el caso $\mathrm{O}_{x}$ en los valores de los rasgos predictores $\mathrm{x}_{i}\left(\mathrm{x}_{i}\left(\mathrm{O}_{0}\right)\right.$ y $\left.\mathrm{x}_{i}\left(\mathrm{O}_{j}\right)\right)$.

ii) Calcular una medida de la semejanza $\delta_{i}\left(\mathrm{x}_{i}\left(\mathrm{O}_{0}\right)\right.$, $\mathrm{x}_{i}(\mathrm{O})$ ) entre estos valores.

Así se define que $\delta$ se obtiene a partir de la siguiente ecuación de distancia euclidiana definida por (4):

$$
\left.\delta_{i}\left(\mathrm{x}_{i}\left(\mathrm{O}_{0}\right), \mathrm{x}_{i}\left(\mathrm{O}_{\nu}\right)\right)\right)=\sqrt{ }\left(\sum\left(\mathrm{x}_{i}(\mathrm{O})-\mathrm{O}_{j}\right)^{2}\right)(4)
$$

Posteriormente se evalúan las distancias entre cada uno de los rasgos predictores y los casos, obteniéndose un rasgo objetivo como respuesta $\beta\left(\mathrm{O}_{0}, \mathrm{O}_{5}\right)$, que es el pronóstico con menor distancia euclidiana o con mayor semejanza.

Finalmente $\mathrm{Y}_{t}\left(\mathrm{O}_{v}\right)$ recibe el valor del indice pronosticado.

\subsection{Algoritmo de predicción UV-B}

Los casos están contenidos en un archivo *csv, de fácil manipulación, pero sin un formato para el procesamiento, entonces se requiere definir la longitud de los rasgos predictores (tamaño del vector), posteriormente se organizan los casos según el tamaño del vector. El presente algoritmo solicita ingresar valores de índice del dia, luego internamente procesa los datos actuales, con los datos de conocimiento referencial y obtiene una respuesta de pronostico UV-B. El algoritmo está contenido en un bucle while loop, para hacer pronósticos continuos hasta presionar 0 para salir.

Código Matlab:

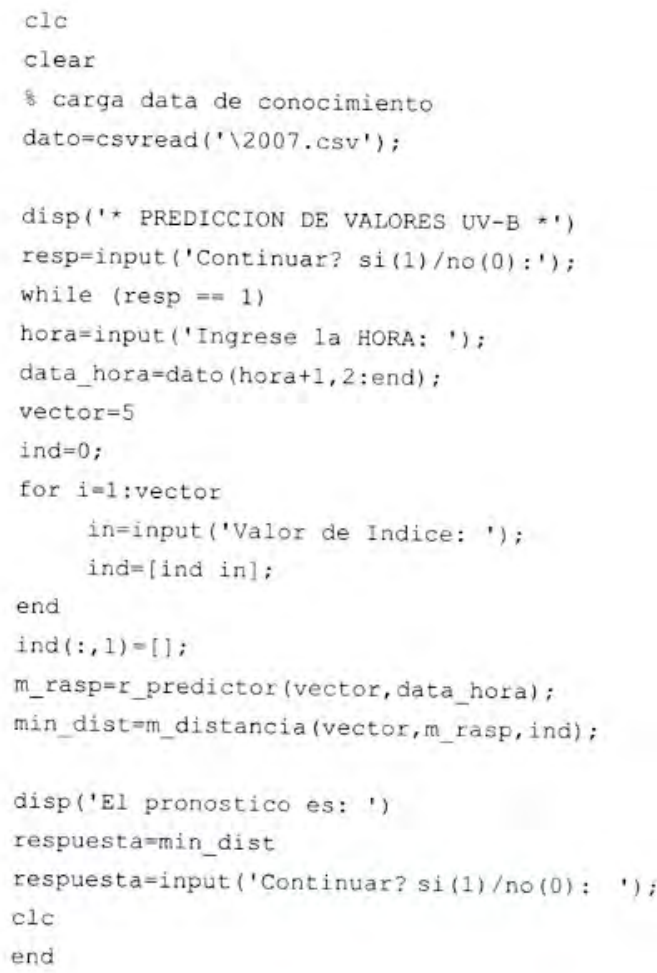

\section{RESULTADOS Y DISCUSIÓN}

Se utilizaron casos de 3 años anteriores, registrados diariamente por el Ministerio de Salud, que forman una parte del conocimiento a consultar, como parte de los rasgos predictores, a fin de obtener el rasgo objetivo (Predicción), obteniéndose lo que se observa en la Figura $\mathrm{N}^{\circ} 01$.

Se obtienen también las curvas de respuesta para los índices de radiación UV-B introducidos y mostrados en la Figura $\mathrm{N}^{\circ} 02$.

Mediante pruebas de eficiencia en respuesta de pronóstico, se probaron diferentes tamaños del rasgo predictor (tamaño de vector), asi se obtuvieron las mejores respuestas con vectores de tamaño 5 a 10 rasgos predictores con 10 pruebas para cada test, como se muestra en la Figura $\mathrm{N}^{\circ} 03$. Donde se observa un promedio de 15 por ciento de diferencia entre el pronóstico y el valor real, es decir aproximadamente más del $85 \%$ de efectividad en el pronóstico.

\section{CONCLUSIONES}

Durante el desarrollo del algoritmo fue necesario tres etapas: Bosquejo, Seudocódigo y Programación. Durante el bosquejo, se entiende el problema a resolver, se consulta la fuente bibliográfica para explicar las variables de 

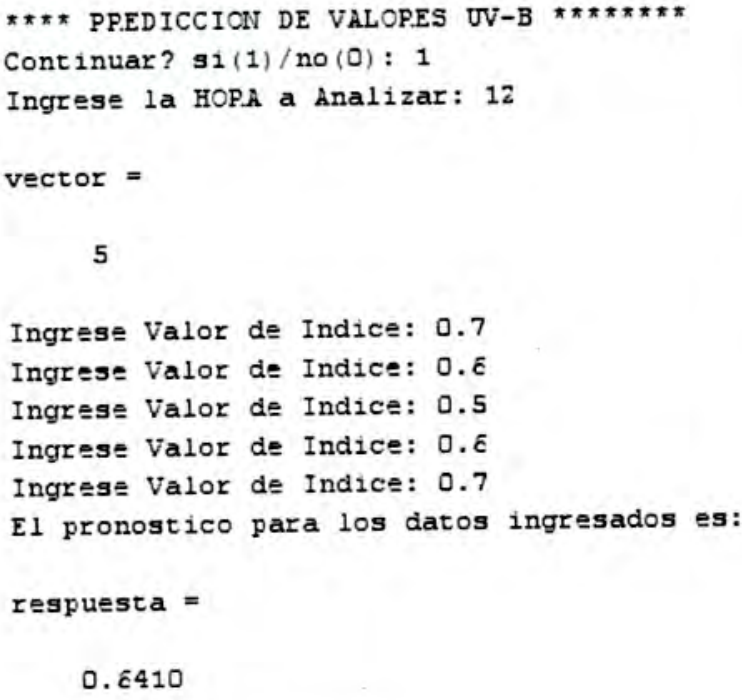

Continuar? si(1)/no(0):

Figura $\mathrm{N}^{\circ} 01$ : Interfaz del usuario Matlab
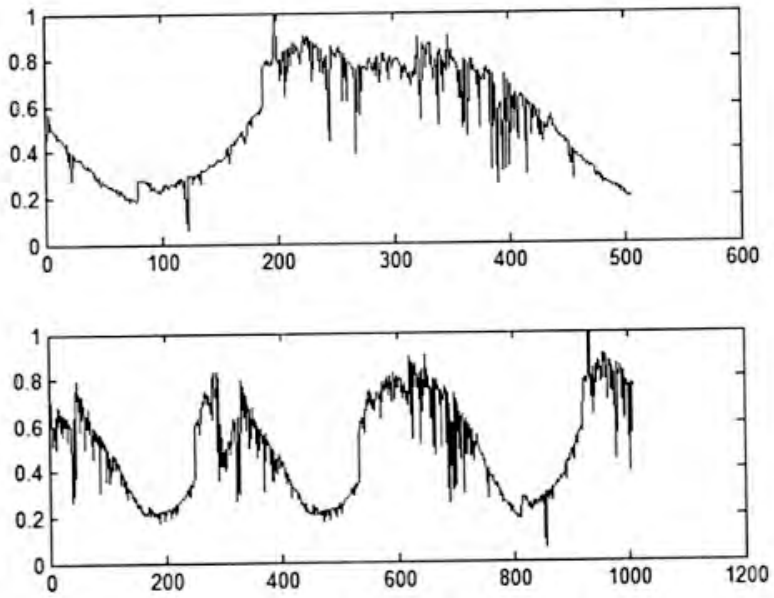

Figura $\mathrm{N}^{\circ} 02$ : Curvas de respuesta entrada sus comportamientos y variables de salida. En la etapa de seudocódigo se migra el problema resuelto en código "borrador", revisando la lógica y secuencia de solución. Finalmente en etapa de programación se implementa en lenguaje Matlab.

Matlab es una herramienta informática de procesamiento. Sirve efectivamente durante el modelamiento de técnicas de predicción y en diversos campos de la investigación científica. Posee numerosas herramientas TOOLBOX capaces de simular diversos escenarios.

$\mathrm{El}$ algoritmo propuesto es una solución posible para analizar los índices de radiación UV-B, en ciudades costeras de Perú.

El presente modelo ofrece respuesta con más del $85 \%$ de efectividad; sin embargo es necesario continuar el estudio de este tipo de algoritmo para ajustar mejores resultados.

\section{REFERENCIAS BIBLIOGRÁFICAS}

[1] Herrera, José, 2013. Tesis Doctoral "Modelo Estocástico a partir de razonamiento basado en casos para la generación de series temporales", Doctorado en Ciencias de la Computación. Universidad Nacional San Agustín. Arequipa-Perú.

[2] Chubachi, S., 1984.Preliminary result of ozone observations at Syowa station from February 1982 to January 1983. Memoir of National Institute of Polar Research, Special Issue 34. In: Proceeding of Sixth Symposium of Polar Meteorology, 13e19.

[3] Longstreth, J., de Gruijlb, F.R., Kripkec, M.L., Abseckd, S., Arnolde, F., Slaperf, H.I., Veldersg, G., Takizawah, Y., van der Leun, J.C., 1998. Health risks, Journal of Photochemistry and Photobiology B 46, 20-39.

[4] M.F. Holick, Evolution and function of vitamin D, Recent Results Cancer Res, 164 (2003)3-28.

[5] UNEP, 2007. UNEP report on environmental effects of ozone depletion, Photochem. Photobiol. Sci. 6, 201-332.

[6] Heisler, G.M., Grant, R.H., Gao, W., Slusser, J.R., 2003.Ultraviolet radiation and its impacts on agriculture

\begin{tabular}{|c|c|c|c|c|c|c|c|c|}
\hline Numero & Tamaño & Vector en & & & & & & \\
\hline Pruebas & 3 & 4 & 5 & 6 & 7 & 8 & 9 & 10 \\
\hline 1.0000 & 4.0189 & 18.3099 & 8.1395 & 6.0000 & 5.4167 & 44.0101 & 24.9084 & 57.2917 \\
\hline 2.0000 & 1.5544 & 7.2000 & 10.3139 & 4.3062 & 7.2539 & 3.1250 & 2.6820 & 22.9773 \\
\hline 3.0000 & 15.3846 & 52.2463 & 177.1930 & 5.2632 & 25.4902 & 36.6142 & 11.2727 & 5.8323 \\
\hline 4.0000 & 5.9859 & 5.4688 & 8.0977 & 14.3639 & 14.1026 & 0.4762 & 5.1230 & 269.5652 \\
\hline 5.0000 & 1.5625 & 3.5014 & 10.2672 & 1.4815 & 20.6897 & 3.6773 & 67.9245 & 7.5853 \\
\hline 6.0000 & 1.3986 & 10.0649 & 0.3984 & 25.4601 & 2.2222 & 14.3695 & 11.9084 & 2.9907 \\
\hline 7.0000 & 16.4804 & 1.8315 & 0 & 3.1000 & 40.9574 & 166.6667 & 9.7744 & 40.7821 \\
\hline 8.0000 & 38.8889 & 11.6667 & 2.2346 & 2.2495 & 2.2727 & 8.2759 & 7.4074 & 4.2654 \\
\hline 9.0000 & 11.8421 & 2.6667 & 0.9317 & 5.8407 & 6.9913 & 31.9149 & 15.0538 & 32.6531 \\
\hline & 28 & 4.8000 & 3.6697 & 25.8065 & 3.7383 & 28.8344 & 10.9155 & 36.4286 \\
\hline
\end{tabular}

promedio $=$

Figura $\mathrm{N}^{\circ} 03$ : Test de algoritmo para diferentes tamaños predictores 
and forests, Agric. For. Meteorol. 120,37.

[7] Caldwell, M.M., Bjorn, L.O., Bornman, J.F., Flint, S.D., Kulandaivelu, G., Teramura, A.H., Tevini, M., 1998. Effects of increased solar ultraviolet radiation on terrestrial ecosystems, J. Photochem. Photobiol. B 46, 40-52.

[8] Kakani, V.G., Reddy, K.R., Zhao, D., Sailaja, K., 2003. Field crop responses to ultraviolet-B radiation, Agric. For.Meteorol.120,191-218. doi:10.1016/j.agrformet.2003.08.015.

[9] OMS, OMM, PNUMA-Organización Mundial de la Salud, Organización Meteorológica Mundial, Comisión de Protección contra la Radiación No Ionizante. Índice UV Solar Mundial: Gua Práctica, Ginebra, Suiza 2003.

[10] A. Osterlind, M.A. Tucker, B.J. Stone, O.M. Jensen, The Danish case control study of cutaneous malignant melanoma. II. Importance of UV-light exposure, Int. J. Cancer 42 (1988) 319-324.
[11] Dr. Alfaro, Cliff, dermatólogo del Hospital de la Solidaridad de Lima. Entrevista RPP, 09-01-2013

[12] SENAMHI Servicio Nacional de Meteorología. Vigilancia de la radiación ultra violeta-B en ciudades importantes del país. Lima, Per, 2012 vol.:8.

[13] Joo F. Escobedo a, Eduardo N. Gomes a, Amauri P. Oliveira b,*, Jacyra Soares b, Modeling hourly and daily fractions of UV, PAR and NIR to global solar radiation under various sky conditions at Botucatu, Brazil, 2008.

[14] Brassard, Gilles; Bratley, Paul (1997), Fundamentos de Algoritmia, Madrid: PRENTICE HALL

[15] DRAE, Vigésima segunda edición

[16] Ferrater, José. Diccionario de filosofa, Ariel, Barcelona, p. 2883.

[17] Cabrera, Sergio S. Radiación Ultravioleta y Salud, Editorial Universitaria, 2005.

\section{Correspondencia:}

Wilson Ferriol Mamani Yanapa: coronel8421@hotmail.com José Herrera Quispe: jherreraq@unsa.edu.pe
Fecha de Recepción: 25/06/2014

Fecha de Aceptación: 09/07/2014 